\title{
Use of empathy in psychiatric practice: constructivist grounded theory study
}

\author{
James Ross and Chris Watling
}

\section{Background}

Psychiatry has faced significant criticism for overreliance on the Diagnostic and Statistical Manual of Mental Disorders (DSM) and medications with purported disregard for empathetic, humanistic interventions.

\section{Aims}

To develop an empirically based qualitative theory explaining how psychiatrists use empathy in day-to-day practice, to inform practice and teaching approaches.

\section{Method}

This study used constructivist grounded theory methodology to ask (a) 'How do psychiatrists understand and use empathetic engagement in the day-to-day practice of psychiatry?' and (b) 'How do psychiatrists learn and teach the skills of empathetic engagement?' The authors interviewed

17 academic psychiatrists and 4 residents and developed a theory by iterative coding of the collected data.

\section{Results}

This constructivist grounded theory of empathetic engagement in psychiatric practice considered three major elements: relational empathy, transactional empathy and instrumental empathy. As one moves from relational empathy through transactional empathy to instrumental empathy, the actions of the psychiatrist become more deliberate and interventional.

\section{Conclusions}

Participants were described by empathy-based interventions which are presented in a theory of 'empathetic engagement'. This is in contrast to a paradigm that sees psychiatry as purely based on neurobiological interventions, with psychotherapy and interpersonal interventions as completely separate activities from day-to-day psychiatric practice.

\section{Declaration of interest \\ None.}

\section{Copyright and usage}

(c) The Royal College of Psychiatrists 2017. This is an open access article distributed under the terms of the Creative Commons Non-Commercial, No Derivatives (CC BY-NC-ND) license.
Despite evidence for the centrality of the doctor-patient relationship in effective psychiatric treatment, ${ }^{1-4}$ the current research paradigm of psychiatry has paid limited attention to the role of empathetic human understanding in routine psychiatric treatment, including in terms of effective methods or techniques for working empathetically with psychiatric patients in routine practice. ${ }^{1,5-7}$ Psychiatry has faced significant criticism in the lay media ${ }^{8}$ and psychiatric literature ${ }^{1,5}$ for overrelying on the Diagnostic and Statistical Manual of Mental Disorders (DSM) and medications while disregarding empathetic, humanistic interventions. ${ }^{1,6,7}$ Helping those with mental illness involves use of empathetic skills throughout practice, including in situations where mental health disorders directly interfere with individuals' willingness to seek help, their ability to form trusting relationships or their ability to communicate with others logically and emotionally. There is increasing neurobiological evidence for the importance of emotional and interpersonal aspects of treatment for patients with mental health conditions. ${ }^{9,10}$ In spite of the evidence for the importance of these empathetic interpersonal aspects of psychiatric treatment, there has been little recent discourse in the psychiatric literature about how empathy can be effectively used in the dayto-day practice of clinical psychiatry. Many academics have written that psychiatry is losing its way as a clinical and academic discipline by overemphasising medication treatments, which often have limited effectiveness, to the exclusion of a deeper, interpersonally rich paradigm of understanding and treating mental illness. ${ }^{1,5,7}$

When empathy or interpersonal interaction with patients is discussed in a psychiatric context, the concept of 'therapeutic alliance' must be considered. Therapeutic alliance is a concept from psychotherapy research, particularly in the psychodynamic tradition, that describes the correlation between a good patienttherapist relationship and good therapy outcomes. Although the therapeutic alliance literature is primarily based on practice within a specific modality of psychotherapy, there is also evidence that therapeutic alliance may influence outcomes in more day-to-day (non-psychotherapeutic) psychiatric practice. ${ }^{3,4}$ Studies of therapeutic alliance have tended to focus on the desirable personal characteristics of the patient and therapist that facilitate a good therapeutic alliance - including 'empathy' on the part of the therapist - with limited elaboration of the specific actions or techniques on the part of the psychotherapist which contribute to this outcome. ${ }^{11-13}$ What is missing from this literature with regard to understanding the use of empathy in psychiatry is an understanding of how empathy as a concept or personal characteristic translates into empathy as a practice in psychiatry.

\section{Understanding empathy}

It is generally accepted that the modern English term 'empathy' is derived from the German word Einfuhlung, which is a term from aesthetics, approximately meaning to 'feel into' an object. ${ }^{14-17}$ Empathy has been studied extensively as a human capacity in a neurobiological context, with significant recent evolution of knowledge, including the description of mirror neurons in the prefrontal cortex that are believed to exist in order to facilitate empathetic resonance with others. ${ }^{18}$ Recent neurobiological attachment models of interpersonal interaction and affect regulation posit, with some evidence, that right-sided structures in the brain moderate affect regulation, which develops and manifests nonconsciously in an empathetic (or non-empathetic) interpersonal context. ${ }^{10}$ There have been varying definitions of empathy in an interpersonal context. Carl Rogers is perhaps most well known for his description of empathy in a mental health context. He defined empathy as 'To perceive the internal frame of reference of another with accuracy, and with emotional components which pertain 
thereto, as if one were the other person, but without ever losing the "as if" condition'. 14,16

In Rogers' theory of optimal patient care in the context of therapy, the therapist's role is to awaken the patient's capacity for growth through the creation of an environment in which he or she feels understood. Rogers, in fact, felt that making a diagnosis is counter-therapeutic and the empathetic understanding is therapeutic in itself, rather than an avenue to understanding the patient so that treatment can be implemented. ${ }^{14,16}$ Barrett-Lennard ${ }^{15}$ describes an 'empathy cycle' in which: a therapist (or responder) listens openly to the patient (or one who is expressing themselves); the therapist resonates with that experience; the therapist expresses the resonance back and then the patient becomes aware of being understood and continues to express her or himself with a heightened level of comfort.

\section{Empathy in clinical medicine}

In the general medical literature - where empathy has been more widely studied than in psychiatry ${ }^{19,20}$ - empathy is widely endorsed as a desirable quality in doctors, and it is correlated with better patient satisfaction, outcomes and savings in time and expense. ${ }^{17}$ It has, however, proven challenging to measure empathy in medical practice, or even to define it satisfactorily. ${ }^{19,20}$ Nonetheless, there is some agreement that clinical empathy involves understanding a patient's perspective, resonating emotionally and expressing caring and concern, sometimes referred to as the cognitive, affective and behavioural dimensions of empathy. ${ }^{21}$ Some argue that empathy is implicitly undervalued in the practice of medicine, especially as modelled in clinical practice, in spite of efforts to teach empathic skills. ${ }^{17,22}$

Several researchers in general medicine have tried to enhance the training of empathy for medical students and residents. The Consultation and Relational Empathy (CARE) scale in general medicine, ${ }^{23}$ which draws on similar concepts to the empathy cycle of Barrett-Lennard, describes and rates basic empathetic interactions in a clinical setting, particularly while listening to patients. One model for teaching empathy to medical students and internal medicine residents combined discussion of why empathy is important with strategising about how to increase empathy in patient interactions, then role playing of clinical scenarios; learner feedback was positive, and learners' confidence in their ability to use empathy skills in clinical practice increased. ${ }^{24}$ Such teaching and evaluation models could provide a framework for empathetic interaction while having relatively straightforward discussions with general medical patients but do not shed much light on how one can interact empathetically when dealing with the interpersonal complexity that psychiatric patients can present.

\section{Empathy in the psychotherapy literature}

In the psychotherapy literature, there is a significant amount of anecdotal and philosophical writing about interactions with patients. Historically, ideas about empathy in psychotherapy have encompassed a tension between empathy as a means of treating the patient and empathy being a means of understanding the patient to optimise treatment. ${ }^{14}$ Much of the psychoanalytic literature emphasises the therapist understanding and influencing the patient within the process of therapy and hence providing a corrective emotional experience, perhaps even allowing the patient to undergo development that was not facilitated in childhood. ${ }^{14,25}$ Marguiles $^{26}$ advocated for the 'empathic imagination' of the therapist, in which understanding and relating to a patient in therapy is a continual creative process in which a pure intersubjective space between the therapist and the patient is created and routinisation or categorisation is actively resisted.

\section{Empathy in general psychiatry}

In general psychiatry, research attention to empathy has been very limited. A recent qualitative study found that resonating with a person and being available to understand them remains a key element of treatment according to patients, ${ }^{2}$ whereas a recent survey-based study found that psychiatrists often rate themselves more highly than their patients do on scales measuring empathy. ${ }^{27}$ But other than the few extant anecdotal accounts, ${ }^{28}$ there has been little empirical work on empathy in general psychiatric practice, particularly in terms of how empathy is enacted. This research gap is problematic, because empathy is especially important and complex to utilise in general psychiatry. Without an understanding of how empathy is enacted and used during the daily tasks of the psychiatrist, empathy remains an abstract concept, rather than a practice that can be discussed, improved and taught effectively.

The lack of a working model for understanding empathy in psychiatric practice when not fully practising a specific modality of psychotherapy poses significant challenges for psychiatric education and the future of psychiatry, because there is little common language to discuss and learn empathy-related skills with psychiatric patients in day-to-day practice. ${ }^{1,5-7}$

In this study, we aimed to describe and better understand how psychiatrists use empathy with their patients in day-to-day clinical practice, while performing routine tasks such as making a diagnosis and providing treatment that might include medications, therapeutic support or involuntary treatment. We aimed to answer the following questions: 'How do psychiatrists understand and use empathetic engagement in the day-to-day practice of psychiatry?' and 'How do psychiatrists learn and teach the skills of empathetic engagement?'

\section{Method}

We used constructivist grounded theory methodology ${ }^{29}$ for our research, consistent with criteria for quality of qualitative research as outlined in Charmaz ${ }^{29}$ and Elliott et $a^{30}$ and as applied in recent medical education literature. ${ }^{31}$ Inductive exploration of a social process (in this case by semi-structured interviewing) and creation of an explanatory, descriptive theory based on the data collected characterise grounded theory in the constructivist grounded theory approach espoused by Charmaz. ${ }^{29}$ Constructivist grounded theory assumes that the social phenomenon in question is interpersonally constructed and contingent upon context. The theory that results from this study is a 'co-constructed' theory between the researchers and the participants. ${ }^{29,30,32}$ Constructivist grounded theory aims to develop a conceptual understanding of a phenomenon while embracing differences of opinion or perspective. ${ }^{29}$ By 'theory', we mean a conceptual understanding of a social process that reflects the perspectives of researchers and research participants and that is situated in a particular context. ${ }^{29}$

Grounded theory demands reflexivity; we engaged, throughout the work, in a routine process of reflection on how our own perspectives might shape our developing analysis. Reflexivity also requires sharing key contextual information with readers. The principal researcher (J.R.) is a psychiatrist with an interest in psychotherapy and medical education, whereas his co-author is a neurologist with roles in educational research and leadership. Interviews were approached from a 'naïve' standpoint, ${ }^{29}$ and divergent viewpoints were encouraged where possible from participants. ${ }^{29}$ The principal researcher was able to gain further subjective input from his co-investigator's (C.W.) 'quasi-outsider' viewpoint as a non-psychiatrist physician.

We sent an email to all 75 psychiatrists and 12 senior residents (PGY4 and 5) from the department of psychiatry at one Canadian 
medical school, inviting their participation. We conducted 21 semi-structured interviews -17 with academic psychiatrists who are clinical teachers and 4 with senior psychiatry residents. The psychiatrists interviewed represented a range of practice types, including general in-patient/out-patient, assertive community treatment, consultation, forensic and other specialised practices. These clinicians would see a range of patients with moderate to severe mental health difficulties, including psychotic disorders, mood disorders such as depression or bipolar disorder, anxiety disorders, personality disorders and substance use disorders combined with other significant mental health disorders. Ethics approval was obtained from the institutional research ethics board.

A broad range of psychiatrists responded to the request for participation, which was in keeping with the study's aim to understand empathy in the general practice of psychiatry. As the study progressed, and in keeping with grounded theory methodology, we engaged in theoretical sampling to more fully elaborate our emerging thematic categories. ${ }^{29}$ For example, in our early analysis, we identified that learning the skills of empathetic engagement in residency was a prominent theme, and we reasoned that extending our sampling to include residents would facilitate exploration of this notion. We also iteratively refined the questions asked in interviews as the study progressed, guided by our evolving analysis. Consistent with constructivist grounded theory principles, as elaborated by Charmaz, ${ }^{29}$ the approach to interviewing evolved as data collection and analysis proceeded, ${ }^{29,31}$ and the nature of the questions asked evolved with the results of the study in an iterative fashion. Although we did not explicitly engage in a 'return of findings' exercise with our participants, our iterative approach to interviewing allowed us to repeatedly test the resonance of our evolving analysis with new participants.

Interviews were audiotaped and transcribed verbatim, without identifying data. Transcripts were analysed using an iterative, constant comparative process. We analysed the initial interviews using line-by-line open coding, assigning a theme to each line of the transcript. Once frequently recurring themes were identified, we had discussions and the principal investigator wrote memos identifying emerging themes that could be components of a potential theory. When several key components were identified, we continued to code, identifying how each line fit into a theme or identifying a new theme that had not yet arisen in the theory. NVivo software was used to organise coding once major themes were identified through the process of manual open coding. We continuously refined themes and our understanding of the relationships between them in the larger theory through continuous memo-writing and periodic discussion. ${ }^{29,31}$ This included particular attention to discrepant examples in order to ensure that the developing theory accounted for their occurrence. Data collection continued until theoretical sufficiency was judged to have been reached. ${ }^{29}$ Sufficiency does not indicate that further sampling might not have revealed new information, but rather that we had sufficient data on which to build a conceptual understanding of how empathy was enacted in practice, without 'gaps or leaps of logic'.33

\section{Results}

In our analysis, we focused on how empathy is enacted in practice within the active clinical context, a process we describe as 'empathetic engagement'. Our theory of empathetic engagement in psychiatry encompasses three key components (Fig. 1): relational empathy, transactional empathy and instrumental empathy. These components of empathetic engagement could be thought of as modes of relating empathetically. Relational empathy describes therapeutic processes that take place when the psychiatrist and the patient are working together towards a common goal and are comfortable with each other. Transactional empathy concerns discussions and activities that arise when there are differences of perspective between the psychiatrist and the patient that must be negotiated collaboratively for the patient to feel engaged and participate in treatment. Instrumental empathy describes activities that the psychiatrist carries out, often using advanced knowledge, in order to understand what is happening when a patient is not cooperative with treatment or is not engaged in a therapeutic relationship with the psychiatrist, particularly when this inability of the patient and psychiatrist to work together stems from the patient's mental health problems.

All of these activities would require the psychiatrist to apply emotional and cognitive understanding of the patient's perspective. As one moves from relational empathy through transactional empathy to instrumental empathy, the actions of the psychiatrist would be more deliberate and interventionist. The theory is not purely developmental; expert practitioners would use skills from all areas of the theory, applying them as necessary based on the situation and the particular patient with whom he or she is

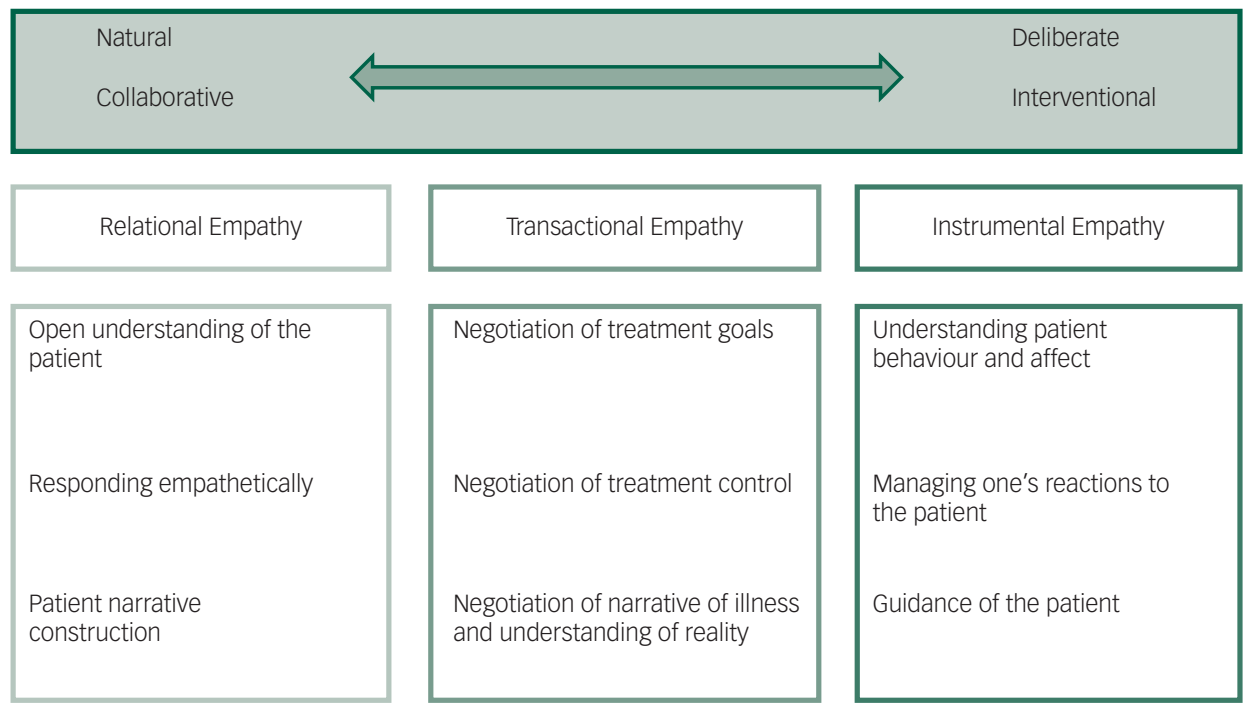

Fig. 1 Components of empathetic engagement with sub-components. 
interacting. There are many patient care activities that overlap these different modes of empathetic engagement and blur the distinction between them (Fig. 2); activities in one mode could be used to facilitate success in another mode.

Each element of this theory is elaborated below, using illustrative quotations from study participants $(\mathrm{P})$.

\section{Relational empathy}

Relational empathy describes those activities that occur when the patient and psychiatrist are communicating effectively. The psychiatrist uses his or her ability to relate to the patient and understand what is going on from his or her perspective as the patient actively seeks to communicate this to the psychiatrist. We identified three core elements of relational empathy from our data: open understanding of the patient, responding empathetically and patient narrative construction.

\section{Open understanding of the patient}

Open understanding of the patient comprised listening to the patient and trying to "put ourselves in their shoes and trying to imagine at least what it would be like to go through what they're going through' (P12), particularly in terms of the meaning of the illness and the events leading up to the presentation. Most participants endorsed the value of these efforts as a foundation for the therapeutic work that they would need to undertake with their patients:

..sometimes the patients are not very comfortable talking about everything, and for them to be able to open up to the physician...it's important they feel safe, and they feel understood, they feel that other person is here with full attention, and is listening, and is willing to see them. (P7)

Participants stressed the importance of not prematurely fitting the patient into predetermined ideas of illness, '...maintaining open-mindedness that you don't know everything when you're walking in the room (P17)', because each person's experience of life and their illness is unique.

\section{Responding empathetically}

Participants emphasised the importance of verbally or nonverbally communicating back to the patient that what they have been expressing has been understood, 'letting them know that you're listening to them and that you're acknowledging what they're saying, you're validating what they're saying...' (P19).

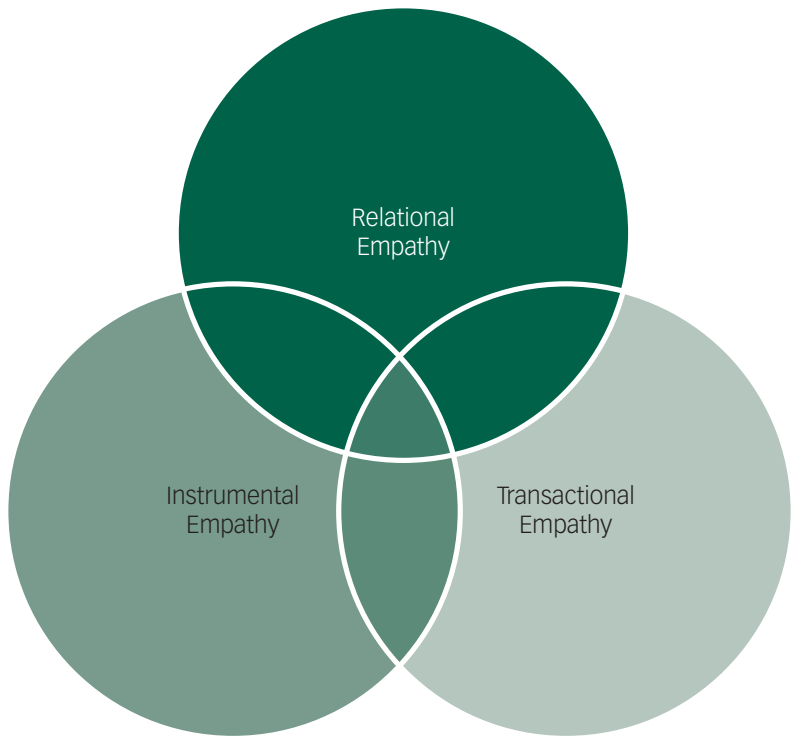

Fig. 2 Relationship of the different components of empathetic engagement.
Participants found value in a 'conversational' approach in which they were 'always trying to deepen the understanding of the material...feeding back to them what I'm hearing, what I'm understanding, seeing if they can enlarge on it and get a picture, the bigger picture' (P18). In this way, relational empathy could sharpen understanding of the patient's story, for the mutual benefit of psychiatrist and patient.

\section{Patient narrative construction}

Beyond listening to the patient and communicating one's understanding back to the patient, participants reported working with patients to find meaning in their own experiences by helping them develop a more coherent narrative of the life experiences that contribute to their presentation.

... a coherent narrative identity, a story to account for what you've been through, how it has affected you, who you are, and what are you capable of doing in the future... I think really dictates, then, what you're willing to do in terms of seeking help, what will recovery or wellness look like for you, and you gain as a clinician the understanding of their language, what's meaningful to them, what's important to them.... (P2)

Although the development of a coherent narrative may serve a diagnostic function for the psychiatrist, this process often reflects instead a human understanding of a difficult situation, because

'if you're ignoring the fundamental understanding of how the patient views themselves in the world... you're not really getting anything about the patient that's meaningful or important to understand how they function in the world' (P6).

\section{Transactional empathy}

Participants described the need to negotiate and to compromise with patients about various aspects of care to facilitate engagement in treatment. This process demands that the psychiatrist actively work towards finding common ground with his or her patient based on an understanding of the patient's subjective viewpoint. Patients' engagement can be facilitated in this mode, 'by trying to provide them with options so that they feel some sense of control' (P19). These negotiated aspects can vary from basic issues that one might find in any area of medicine such as the goals of treatment and the diagnosis to the relationship with the psychiatrist and the treatment plan. As described below, it may also enter into areas more specific to psychiatry such as the negotiation of ward privileges or the shared understanding of reality itself.

\section{Negotiation of treatment goals}

When seeing a patient for the first time and on an ongoing basis throughout treatment, participants described a need to develop a shared understanding with the patient of the diagnosis and the goals of treatment. To reach this shared understanding, participants described listening to what the patient said and trying to understand him or her and reach a compromise while respecting the patient's autonomy.

And, I think that I try very hard to let people identify things that are salient to them, so things that they want to work on, that they want to target, that they see as issues, so that we can kind of come to a shared conclusion about what we're going to go after and make up some goals together. (P8)

\section{Negotiation of the role of the psychiatrist and the treatment plan}

Participants described that the role that the psychiatrist played with the patient, the nature of the treatment and the parameters of treatment were often called into question, requiring negotiation. As one participant described, when one is a patient '...coming into a hospital and there are certain rules and expectations... there's a certain degree of anxiety or trepidation that most people would feel entering such a relationship' (P17). Understanding the position participants' patients were in, including recognising the fear they might feel seeing a psychiatrist, was described as 
something that could bolster the process of negotiation. Patient care in psychiatry can involve temporary loss of autonomy, and compromising with patients on day-to-day issues such as ward privileges was seen by participants as a way of engaging them in treatment. In one interview, a psychiatrist describes an interaction with a young woman who had symptoms of mania:

she's really bored... She's okay being in the hospital for now, but she wanted passes to the ward for as long as she wants, and with cards, and she wanted to have her phone at all times. And, so after some discussions and collaboration, we came up with the plan that, instead, she would have the phone for half-an-hour intervals throughout the day. It's got her less bored, and it really calmed her down momentarily. (P1)

\section{Negotiation of narrative of illness and understanding of reality}

Participants reported that when working with complex or severe patients, compromises about the shared understanding of the nature of reality itself - particularly in the context of the patient's experiences - were sometimes productive. As an example, one participant described such a compromise made in her work with a patient who had significant psychosis symptoms:

I think it led to a therapeutic outcome, imagining tryin to relate in some way. I mean this is a person who lives in a completely different world than I do but by putting an effort into imagining what that world must be like, I think it helped. (P12)

Participants described that initially compromising on the common understanding of reality might facilitate the clinical process and eventually help the patient to appreciate the influence of their illness on their view of reality:

The second time I met her, she was more open to that [understanding of psychosis]. The third time... she was like, I can't believe you had to listen to me. That whole interview was just talking about all this bizarre stuff, and you just listened and you were just there... It was interesting because she was almost trying to empathise with me and what it was like to listen to her. (P2)

\section{Instrumental empathy}

Participants reported facing situations in which advanced skills were required to understand and manage a patient's behaviours and to navigate the relationship between psychiatrist and patient These advanced skills, which we have termed instrumental empathy', involve efforts to understand the behaviour and actions of the patient, even when the patient himself or herself is not fully able to do so. As one participant noted, 'if somebody is in a very acute situation, extremely kind of unstable, maybe [with severe psychosis symptoms] for example, you still need to understand them, but it needs to be done in a different way...' (P19). Participants reported drawing on theoretical constructs from psychotherapy and psychiatric diagnosis, while grounding these concepts in empathetic understanding, in order to develop strategies for managing challenging patients, as we describe below.

\section{Understanding patient behaviour and affect}

Participants recognised the value of identifying a patient's psychological mechanisms such as defences. Developing an understanding of patients' behaviour was facilitated, many felt, by using emotional and non-verbal cues 'to read people, and read between the lines' (P15). Engagement and empathetic treatment were facilitated by an advanced understanding of patients' pathology and what the experience of it might be like:

If I thought that... you were locking me up and you were going to hurt me or hurt my family, I would not be speaking kindly to you, and I would be trying to bolt from the ward. So it makes perfect sense to me that, since... they're believing that the world is going to come to an end while I have them locked up, that they would be yelling, screaming, and fighting with the nurses. (P9)

\section{Managing one's reactions to the patient}

Participants used their awareness of the defences and motivations underpinning their patients' behaviour to modulate the doctorpatient relationship. At these times, one has to 'be a little more thoughtful, you have to say, okay, they're coming at me, they're mad at me, they're whatever... let's kind of re-approach this, let's do this a little bit differently' (P15). Psychiatrists described using their empathetic skills to know how to intervene in the treatment relationship while maintaining the patient's sense of feeling engaged and cared for. In cases when the patient was hostile or lacked awareness of the reasons for their opposition to a treatment plan, participants found it helpful to use dialogue or their own reactions to the patient to figure out the nature of the problem. They described then using this knowledge to manage the therapeutic relationship, either through reacting differently to the patient or through sensitive confrontation of the issue. Recognising this element of instrumental empathy in his or her treatment of patients experiencing personality disorder-related symptoms, one participant noted,

I see a lot of people who are what I would call help rejecters their way of developing connection is to have a chaotic inconsistent connection. So I can't expect their connection to be the way that maybe I develop connection... So if I felt a certain way about a certain interaction, what part of that was me? What part of that was the patient or the interpersonal interaction? ...I think that's helped me learn about those kinds of experiences. (P14)

\section{Guidance of the patient}

Instrumental empathy allowed participants to use their efforts to understand the patient's difficulties and their experiences to develop a treatment approach that they hoped would be both beneficial and aligned to the patient's needs, goals and wishes. Participants described sometimes needing to implement 'empathy in a tough love [or] limit type of approach' (P16) which could seem insensitive to the patient's immediate or stated desires but that was more in keeping with their longer-term treatment needs. Interventions grounded in the understanding gained through empathetic engagement such as setting boundaries, creating a therapeutic experience or changing the terms of the treatment so that a patient's psychological mechanisms interfere less with it were discussed. These actions were perceived by the clinician to benefit patients in the long run:

I can't approach it from a sympathetic point of view for the most part because she explodes on people who are sympathetic towards her because she views it as patronising... So, sympathy doesn't work. In fact with her it's much more of a paternalistic here's what you're going to do and here's why, and explaining it. It's a very different approach but it helps manage with her symptoms. (P10)

\section{Discussion}

Our grounded theory makes visible the tacit practices of empathy in clinical practice by psychiatrists, which we have called 'empathetic engagement'. With the notion of empathetic engagement, we move beyond empathy as an abstract idea, describing a set of active empathy-driven practices that clinicians use in situ. Our theory of empathetic engagement reflects elements consistently described by many of those who were interviewed. This theory and its attendant language provide a means to conceptualise the use of empathy in clinical psychiatric practice for discussion and educational purposes.

The theory presented in this paper outlines interactions with patients that use empathy as a skill in clinical practice, or empathetic engagement, to address a range of patient needs in a variety of clinically relevant situations. Relational empathy could be used with most patients in order to listen to the patient, understand the patient, collaboratively formulate a narrative of their problems and reflect back to the patient that they have been understood, while hopefully facilitating better self-understanding in the patient. Transactional empathy could arise in many situations with different types of patients in situations ranging from discussing treatment options with a patient who is experiencing panic to softening the 
impact of having to involuntarily admit a patient who has psychosis symptoms that put his or her life at risk. Instrumental empathy could come into play when patients are having complex behavioural issues stemming from disorders that affect their ability to understand why they are having such behaviours. Severe personality disorders, substance use disorders and psychotic disorders are all examples which could be applicable. This study presents a preliminary theory, but further discussions or investigation of empathetic engagement could facilitate understanding of how these practices could enhance treatment for patients with different disorders of varying severity.

It is necessary to create categories in order to conceptualise a theory for discussion but the different categories of empathetic engagement blend together (Fig. 2). For example, in a single therapeutic discussion with a patient experiencing suicidal thinking, it is conceivable that one might use relational empathy to understand why the person is upset (including if it is for reasons that are incompatible with the treater's perception of reality), one might use instrumental empathy to understand how one's reactions may upset or calm the patient and to act in a corresponding manner and one might use transactional empathy to try to understand what the patient wants and to collaboratively reach a treatment plan balancing safety and the patient's autonomy.

\section{Relative absence of a discourse of empathy in the psychiatry paradigm}

Our theory addresses a need, which Bracken et al highlighted, to 'develop a different sensibility towards mental illness itself and a different understanding of our role as doctors'. In fact, the ease with which participants in our study engaged with ideas about empathy and elaborated specific empathy-influenced practices suggests that psychiatry, as actually practiced, is not so lacking in empathy or humanism as some critiques ${ }^{8}$ would have us believe. What has perhaps been lacking is a language that renders these practices visible and makes them available for conversations with learners and colleagues.

As Pedersen ${ }^{19}$ points out, it is more productive to talk about empathy as embedded within biomedical acts than as something additional to them. It is perhaps the fact of empathy being tacitly embedded within psychiatry that has made it so difficult to talk about and study. We propose that it is more productive to discuss 'empathetic engagement' as a situated practice as opposed to 'empathy', which is often seen as an abstract concept or a complementary activity, for the enhancement of the psychiatric discourse as it relates to empathetic patient care.

\section{Relation to the therapeutic alliance literature}

The psychotherapeutic therapeutic alliance literature describes the patient and physician factors that contribute to a good working relationship in psychotherapy or in psychiatric follow-up. A good therapeutic alliance has a positive effect on treatment outcomes. ${ }^{3,4,11,13}$ Our notions of transactional empathy and instrumental empathy parallel the therapeutic alliance literature's emphasis on recognising and interpreting non-verbal communication and on attending to and repairing ruptures in the therapeutic alliance. ${ }^{13}$ Although there are structured methods for recognising and interpreting non-verbal communication and attending to alliance ruptures that are understood in psychodynamic psychotherapy, ${ }^{34}$ this paradigm of understanding is not universally applicable in general psychiatric practice, especially with patients who have more complex pathology, which is often not amenable to purely psychodynamic interventions. Our theory extends the understanding of the interaction between psychiatrist and patient beyond the realm of practice within a specific modality of psychotherapy by describing how empathetic practices, including psychotherapy skills, can be applied throughout the practice of day-to-day psychiatry in different situations and settings.

\section{Relation to the empathy literature in general medicine}

Neither the current therapeutic alliance literature nor the empathy literature in medicine ${ }^{19,20}$ has managed to fully describe how empathy is enacted in the situations that arise within practice. The descriptive language of 'empathetic engagement' we present in this study, as a practice in situ, in contrast to the more abstract ideas of empathy that dominate the literature, could contribute to discussion of these aspects of practice.

Much of the general medical literature on physician empathy has attempted to measure empathy using scales. The Jefferson Scale of Physician Empathy, ${ }^{21}$ for example, was specifically designed to test empathy in physicians and is one of the scales most frequently used to measure empathy in doctors. The Jefferson scale is one of the best empirically supported measures, but its self-report questions are mostly about attitudes outside of any true clinical context and the questions are fairly transparent, and as Hemmerdinger et $a l^{20}$ describe, it is easy for practitioners to 'cheat the test'. Furthermore, as Pedersen also argues, in line with poststructuralist cultural criticism, ${ }^{35}$ those aspects of a clinical encounter that can be reified into measurable variables do not always capture the most important elements of an interpersonal encounter. Our theory, by describing the enactment of embedded empathetic psychiatric practices, hopefully provides some language through which these can be discussed, developed and taught.

Our theory, particularly relational empathy, bears some similarity to existing constructs in the medical empathy literature. Our account of relational empathy is very similar to the pre-existing construct of relational empathy used in the CARE scale in general medicine $e^{23}$ and the empathy cycle of Barrett-Lennard. ${ }^{15}$ The term 'transactional empathy' is used in the psychodynamic literature from psychiatry in the late 1980s to describe the process through which a patient's unconscious processes are elucidated and processed by a transaction with the therapist but this represents a much different construct from that presented in our theory. ${ }^{26}$ The absence in our theory of the differentiation between emotional, cognitive and behavioural aspects of empathy that often arise in medical models of empathy might reflect the fact that this theory is based on situated practice rather than more abstract concepts of empathy.

\section{Relation to psychotherapy empathy literature}

Our theory incorporates Rogers' concept of perceiving the internal frame of another. All elements of the theory that we present implicitly incorporate this idea but the shared frame of reference is applied to specific practice-related tasks in psychiatry rather than being an object unto itself. Although Rogers aimed, through therapy, to awaken a capacity for growth in the patient, this goal is less prominent within our theory except throughout relational empathy. Transactional empathy and instrumental empathy aim to facilitate treatment, supporting the resolution of mental health problems. Rogers' opposition to diagnosis, as well as Marguiles,26 idea of unencumbered creative empathy, is not practicable in much of psychiatric practice because many patients seen by psychiatrists need more intervention than such an approach would make possible. Our theory emphasises being as open to the patient's perspective as possible while still being able to intervene medically (including through medical-psychosocial means such as psychotherapy) to help them get better from mental health problems. Our position is that a medical model remains useful and necessary for many individuals who suffer from severe mental health disorders and that increased attention to the empathetic aspects of treatment will optimise the experience and outcomes for our patients. 


\section{Teaching empathy skills}

Our theory was developed with the goal of facilitating discussion and teaching about empathetic practices in psychiatry. A comprehensive discussion of educational theory about teaching interpersonal skills such as empathy and how our theory may apply is beyond the scope of this paper. Researchers in general medicine support the idea that empathy skills can and should be taught in medicine. ${ }^{21,23}$ One model differentiates between dispositional empathy, which is a character trait and cannot be taught, and situational empathy, which are the aspects that can be taught and practiced. $^{23}$ By this definition, our theory could contribute to the discussion and teaching of situational empathy. It is hoped that by providing a language and a framework in which to discuss how empathy is used in psychiatric practice, we have also created a starting point for teaching it clinically.

\section{Limitations}

Being a constructivist theory, there is implicit and express acknowledgement that the researcher brings in his or her own perspective, ${ }^{29,31}$ particularly as an 'insider'. ${ }^{32,36}$ Many of the participants are colleagues of the principal researcher, potentially influencing the responses they provided in interviews. The interview technique, however, involved asking participants to describe specific experiences, rather than asking about empathy in the abstract, grounding the data in participants' own clinical practices and mitigating the influence of the researcher's 'insider status' on the data collected. Regular discussion of the interview data and the emerging theory with the co-investigator, a non-psychiatrist physician, helped to expand the range of ideas and to challenge the principal investigator's assumptions.

Grounded theory cannot aim for generalisable results, the analysis is always influenced by context. ${ }^{29,31}$ Our results are based on a single Canadian academic psychiatry setting. We anticipate, however, that our analysis offers concepts that are transferrable to other settings where psychiatric educators are considering how to effectively discuss and teach 'empathetic engagement'. Other qualitative approaches may have yielded different kinds of insights, but in our view we were exploring what is fundamentally a social process, making the affordances of grounded theory particularly appropriate.

\section{Implications}

We hope that our theory of empathetic engagement can be applied to broader questions of how we engage meaningfully with patients in day-to-day psychiatry and can inspire further research and innovation in psychiatric education, particularly in residency programmes. It appears that many desirable practices are in place but are in danger of being marginalised over time unless they are codified through research and development at an academic level. ${ }^{1,5-7,14}$ Despite the absence of discourse on empathetic practices in contemporary academic psychiatry, we have shown that empathy-based approaches actually permeate psychiatric practice. What has been missing is perhaps not so much the practices themselves but the language to allow those practices to be discussed, to be taught and to evolve.

\footnotetext{
James Ross, MD, MHPE, FRCPC, Department of Psychiatry, Western University, Victoria Hospital, London, Ontario, Canada; Chris Watling, MD, PhD, FRCPC, Department of Clinical Neurological Sciences, Western University, London, Ontario, Canada; Centre for Education Research and Innovation (CERI), Western University, London, Ontario, Canada

Correspondence: James Ross, Department of Psychiatry, Western University, Victoria Hospital, London, Ontario, Canada. Email: james.ross@|hsc.on.ca

First received 24 Oct 2016, final revision 22 Dec 2016, accepted 16 Jan 2017
}

\section{Acknowledgements}

Dr Jan Van Dalen from the University of Maastricht reviewed the full thesis from which this manuscript was derived and provided feedback on its contents. Dr Paul Links reviewed this manuscript and made suggestions about its contents.

\section{Funding}

This project was supported by internal funding from the Western University Department of Psychiatry.

\section{References}

1 Bracken P, Thomas P, Timimi S, Asen E, Behr G, Beuster C, et al. Psychiatry beyond the current paradigm. Br J Psychiatry 2012; 201: 430-4.

2 Johansson $\mathrm{H}$, Eklund M. Patients' opinion on what constitutes good psychiatric care. Scand J Caring Sci 2003; 17: 339-46.

3 Frank AF, Gunderson JG. The role of the therapeutic alliance in the treatment of schizophrenia: relation to course and outcome. Arch Gen Psychiatry 1990; 47: 228-36.

4 Johansson $\mathrm{H}$, Jansson J. Therapeutic alliance and outcome in routine psychiatric outpatient treatment: patient factors and outcome. Psychol Psychother 2010; 83: 193-206.

5 Kleinman A. Rebalancing academic psychiatry: why it needs to happen - and soon. Br J Psychiatry 2012; 201: 421-2.

6 Baldessarini RJ. The impact of psychopharmacology on contemporary psychiatry. Can J Psychiatry 2014; 59: 401-5.

7 Joober R. Deconstructing the mental health crisis in only 2 pieces. J Psychiatry Neurosci 2016; 41: 222-4.

8 Angell M. The illusions of psychiatry. New York Review of Books 14 Jul 2011 (http://www.nybooks.com/articles/2011/07/14/illusions-of-psychiatry/).

9 Gabbard GO. Textbook of Psychotherapeutic Treatments. American Psychiatric Publishing, 2009

10 Schore A. The right brain is dominant in psychotherapy. Psychotherapy 2014; 51 388-97.

11 Ackerman SJ, Hilsenroth MJ. A review of therapist characteristics and techniques positively impacting the therapeutic alliance. Clin Psychol Rev 2003; 23: 1-33.

12 Horvath $\mathrm{AO}$. The therapeutic relationship: research and theory. Psychother Res 2005; 15: 3-7

13 Summers R, Barber J. Therapeutic alliance as a measurable psychotherapy skill. Acad Psychiatry 2003; 27: 160-5.

14 Bennet MJ. The Empathic Healer: An Endangered Species? Academic Press, 2001

15 Barrett-Lennard GT. The empathy cycle: refinement of a nuclear concept. J Couns Psychol 1981; 28: 91-100

16 Tudor K. Understanding empathy. Transactional Anal J 2011; 41: 39-57.

17 Mercer SW, Reynolds WJ. Empathy and quality of care. Br J Gen Pract 2002; 52 Suppl: S9-12.

18 Young A. Empathy, evolution and human nature. In Empathy from Bench to Bedside (ed. J Decety): 21-37. MIT Press, 2012

19 Pedersen R. Empirical research on empathy in medicine - a critical review. Patient Educ Couns 2009; 76: 307-22.

20 Hemmerdinger JM, Stoddart SDR, Lilford RJ. A systematic review of tests of empathy in medicine. BMC Med Educ 2007; 7: 24

21 Hojat M, Mangione S, Nasca TJ, Cohen MJM, Gonella JS, Erdman JB, et al. The Jefferson scale of physician empathy: development and preliminary psychometric data. Educ Psychol Meas 2001; 61: 349-65.

22 Pedersen R. Empathy development in medical education - a critical review. Med Teach 2009: 32: 593-600.

23 Hanzevacki M, Jakovina T, Bajic Z, Tomac A, Mercer, S. Reliability and validity of the Croatian version of Consultation and Relational Empathy (CARE) measure in primary care setting. Croat Med J 2015; 56: 50-6.

24 Aggarwal R, Guanci N. Teaching empathy during clerkship and residency. Acad Psychiatry 2014; 38: 506-8.

25 Emde RN. Mobilizing fundamental modes of development: empathic availability and therapeutic action. J Am Psychoanal Assoc 1990; 38: 881-913.

26 Marguiles A. The Empathic Imagination. WW Norton, 1989.

27 Aggarwal R. Empathy: do psychiatrists and patients agree? Am J Psychiatry Residents J 2007; 2: 2-3. 
28 Faden J. Maintaining empathy in a locked psychiatric unit. J Am Osteopath ASSOC 2013; 113: 364-5.

29 Charmaz K. Constructing Grounded Theory. Sage, 2006.

30 Elliott R, Fischer CT, Rennie DL. Evolving guidelines for publication of qualitative research studies in psychology and related fields. Br J Clin Psychol 1999; 38: 215-29.

31 Watling C, Lingard L. Grounded theory in medical education research: AMEE guide no. 70. Med Teach 2012; 34: 850-61.

32 Morrow SL. Quality and trustworthiness in qualitative research in counselling psychology. J Couns Psychol 2005; 52: 250-60.

33 Morse JM. The significance of saturation. Qual Health Res 1995; 5: 147-9.
34 Gabbard GO. Psychodynamic Psychotherapy in Clinical Practice (3rd edn). American Psychiatric Publishing, 2005.

35 Latour B. We Have Never Been Modern (trans C Porter). Harvard University Press, 1991.

36 Dwyer S, Buckle J. The space between: on being an insider-outsider in qualitative research. Int J Qual Methods 2009; 8: 54-63. 